\title{
Atomwärme, Volumelastizität und Eigenfrequenz bei einatomigen Metallen \\ von
}

A. L. Bernoulli.

(31. VIII. 18.)

Der erste Forscher, welchem es gelang, optische Konstanten aus elastischen Daten zu berechnen, war E. Madelung ${ }^{1}$ ). Für mehrere Halogenide der Alkalien berechnete er auf Grund der folgenden an Hand eines geeigneten Modells hergeleiteten Gleichung die Schwingungszahlen $\nu$ der Reststrahlen des betreffenden Materials aus den Kompressibilitätskoëffizienten $x$, also aus der Volumelastizität, wobei $\varrho$ die Dichte und $\mathrm{M}_{1}$ und $\mathrm{M}_{2}$ die Atomgewichte der die Verbindung bildenden beiden Elemente bedeuten. Nach Madelung ist die Beziehung erfüllt:

$$
\nu=1,709 \cdot 10^{i} \frac{\sqrt[3]{\left(M_{1}+M_{2}\right)^{2}}}{\sqrt[6]{\varrho} \sqrt{\mathrm{M}_{1} \cdot \mathrm{M}_{2}}} \sqrt{\frac{\mathrm{C}}{\varkappa}}
$$

Die Grössenordnung ergab sich richtig zu $10^{12}$ Schwingungen pro Sekunde, und wenn auf Grund der als bekannt vorausgesetzten Wellenlänge des Steinsalzes der Faktor C von der Grössenordnung der Einheit gleich 2,24 gesetzt wurde, ergab sich eine bemerkenswerte Übereinstimmung zwischen Theorie und Versuch namentlich für Kaliumchlorid und Kaliumjodid. Unabhängig davon hat A. Einstein ${ }^{2}$ ) im folgenden Jahre 1911 für einen homogenen festen Körper die Beziehung abgeleitet

$$
\lambda=1,08 \cdot 10^{3} \cdot M^{1 / 3} \cdot \varrho^{1 / 6} \cdot \varkappa^{1 / 2}
$$

für die Wellenlängen $\lambda$, welche die Schwingungszahlen, die die Einstein'sche Theorie der spezifischen Wärmen voraussetzt, mit der Kompressionskonstante $x$ in Beziehung bringt. Auch hier ist die Übereinstimmung eine bemerkenswerte. Man erkennt sofort, dass für den speziellen Fall $M_{1}$ gleich $M_{2}$ beide Gleichungen bis auf einen Faktor von der Grössenordnung der Einheit identisch werden, obschon die Prämissen, welche zur Herleitung der
1) Ph. Z. 11, 898 (1910).
2) W. [4] 34, 170 (1911). 
beiden Gleichungen dienten, wesentlich verschiedene sind. Als Ausgangspunkt seiner Theorie der spezifischen Wärmen diente bekanntlich $P$. Debye ${ }^{1}$ ), eine von ihm zuerst abgeleitete neue Beziehung zwischen Kompressibilität und Eigenfrequenz, in welche ausser Molekulargewicht und Dichte noch der Poisson'sche Modul, also allgemein ausgedrückt, neben der Volumelastizität auch noch die Gestaltselastizität eingeht. Die numerische Übereinstimmung zwischen den so berechneten Schwingungszahlen und den nach Debye aus den spezifischen Wärmen berechneten ist da, wo zuverlässige Messungen vorliegen, eine sehr gute. Für den Fall des einatomigen kubischen Kristallgitters ist die Theorie von Born und $v$. Kàrmàn ") gegeben worden, und weiterhin von Thirring ${ }^{3}$ ) auch für zweiatomige kubische Gitter.

So verschiedenartig die Ausgangshypothesen zum Teil bei den genannten Forschern, denen auch $W$. Sutherland ${ }^{4}$ ) sich anreiht, auch sein mögen, alle gelangen zum Resultat, dass die Schwingungszahlen der sechsten Wurzel aus der Dichte und der Quadratwurzel aus der Kompressibilität umgekehrt proportional sind. Einstein ${ }^{5}$ ) hat seine eigene Formel nachträglich bis auf einen Zahlfaktor auch durch eine Dimensionalbetrachtung abgeleitet, während er ursprünglich Anziehungskräfte zwischen der schwingenden Molekel und den ruhendgedachten Nachbarmolekeln eines kubischen Gitters als Ausgangshypothese vorausgesetzt hatte. Auf Schwierigkeiten, welche seiner frühern Auffassung entgegenstehen, hat Einstein selbst ausdrücklich ${ }^{6}$ ) aufmerksam gemacht. Eine vorzügliche Zusammenstellung des bisher Erreichten gibt C.E. Blom ${ }^{7}$.

Es schien mir daher nicht uninteressant, zu versuchen, ob man nicht wenigstens die Einstein'sche Gleichung ohne diese oder andere Hypothesen über Fernkräfte oder über die potentielle Energie der schwingenden Molekeln herleiten könne und zwar derart, dass sich auch der Proportionalitätsfaktor, ohne neue Hypothesen einzuführen, in Übereinstimmung mit der Beobachtung ergeben sollte.

Sei $J$ das Trägheitsmoment und $\omega$ die mittlere Winkelgeschwindigkeit der rotierenden Molekel eines Versuchskörpers der

1) W. [4] 39, 789 (1912). $\quad$ 2) Ph. Z. 13, 297 (1912). 3) Ph. Mag. 20, (6ó7 (1910). 4) Citiert nach Valentiner, Quantenhypothese. Braunschweig 1914, S. 37.5 5) W. [4] 35, 679 (1911). 6) W. [4] 42, 1397 (1913). 7) l. c. . 683. 
im Kubikzentimeter $\mathbf{n}$ Molekeln enthält, so ist die kinetische Energie der Rotation aller Molekeln im Kubikzentimeter gegeben durch $\mathrm{H}=\mathbf{n} \cdot \mathbf{J} \cdot \frac{\omega^{2}}{2}$. Wird jetzt der Probekörper durch einen allseitigen äussern Druck komprimiert, so werden die Schwerpunkte der Molekeln gegen die Rotationsachsen der Molekel verschoben. Für eine Molekel, deren Achse senkrecht zur Resultante s des Schubes liegt, wird, da die Achse sich relativ zum Molekelschwerpunkt demnach um die Strecke s verschoben hat, das neue momentane Trägheitsmoment um den Betrag von $\mathrm{ms}^{2}$ grösser sein als vorher, da nach dem Steiner'schen Satze das Trägheitsmoment um eine beliebige Achse gleich ist demjenigen um eine parallele Achse durch den Schwerpunkt, vermehrt um das Trägheitsmoment $\mathrm{ms}^{2}$ eines Massenpunkts $\mathrm{m}$ im Schwerpunkt inbezug auf die neue, um die Strecke s abliegende parallele Achse. Bleibt die mittlere Tourenzahl durch die Kompression ungeändert, so ist der Zuwachs an kinetischer Energie W, den der gepresste Körper pro Kubikzentimeter durch die Kompression instantan erfährt, gegeben durch

$$
\mathrm{W}=\frac{\omega^{2}}{2} \sum_{1}^{\mathrm{n}} \overline{\mathrm{ms}^{2}}
$$

Wir denken uns ferner, um die Rechnung zu vereinfachen, den aus $\mathbf{n}$ gleichen Molekeln mit beliebig im Raum orientierten Drehachsen bestehenden Probekörper ersetzt durch einen solchen mit gerichteten Achsen, sodass also nunmehr je ein Drittel der Molekel um Achsen parallel zur X-Achse, Y-Achse oder Z-Achse eines nach den Würfelkanten des Probekörpers orientierten Koordinatensystems rotieren soll.

Betrachten wir, um unsere Vorstellungen zu fixieren, eine um die X-Achse rotierende Molekel. Bei der allseitigen Kompression wird ihr Schwerpunkt in der Richtung der Achsen die drei Verschiebungen $\Delta_{\mathrm{x}}, \Delta_{\mathrm{y}}, \Delta_{\mathrm{z}}$ relativ zu den ursprünglichen Drehachsen erfahren. Die Verschiebung $\Delta_{\mathrm{x}}$ längs der Drehungsachse ändert nichts am Trägheitsmoment, also auch nichts an der kinetischen Energie dieser Molekel, wohl aber tun dies die beiden andern Verschiebungen $\Delta_{y}$ und $\Delta_{z}$. Bezeichnet $\mathrm{W}_{\mathrm{x}}$ den Zuwachs an kinetischer Energie der Rotation, welche die $\mathrm{n} / 3$ um die $\mathrm{X}$ Achse rotierenden Molekel erfahren, so wird diese Grösse

$$
\mathrm{W}_{\mathrm{x}}=1 / 2 \omega^{2} \cdot \frac{\mathrm{n}}{3}\left(\mathrm{~m} \Delta_{\mathrm{y}}{ }^{2}+\mathrm{m} \Delta_{\mathrm{z}}{ }^{2}\right) \text {. }
$$


Adđiert man hiezu die beiden entsprechenden Ausdrücke für die beiden andern Achsen, die durch zyklische Vertauschung sich ergeben, so wird für den Zuwachs $W$, welche die kinetische Rotationsenergie des Probekörpers pro Volumeinheit erfährt, die Beziehung gelten:

$$
\mathrm{W}=1 / 2 \mathrm{~m} \omega^{2} \cdot 2 / 3 \mathrm{n}\left(\Delta_{\mathrm{x}}{ }^{2}+\Delta_{\mathrm{y}}{ }^{2}+\Delta_{\mathrm{z}}{ }^{2}\right) . . .
$$

Für einen isotropen festen Körper, und ebenso natürlich für eine Flüssigkeit, werden die drei Verschiebungskomponenten einander gleich, wodurch

$$
\mathrm{W}=\mathrm{nm} \cdot \omega^{2} \cdot \Delta^{2}
$$

Das Wesentliche an der hier gegebenen Herleitung dieser Gleichung liegt darin, dass wir dabei nicht anzunehmen brauchen, dass zwischen den Molekeln anziehende oder abstossende Kräfte wirksam sind, und ferner dass diese Herleitung keinerlei Annahmen über die Temperaturabhängigkeit der Rotationsenergie enthält.

Damit möchte ich jedoch keineswegs prinzipiell gegen die Annahme derartiger Kräfte etwas einwenden; ich will nur betonen, dass diese Gleichung ohne die Annahme derartiger Kräfte abgeleitet werden kann.

Es bleibt uns nun noch die Aufgabe, den nach Gleichung 1) oder 1a) berechneten Wert der Arbeit $W$ durch die Kompressibilität $x$ und die Volumverminderung $\Omega$, welche der Probekörper pro Kubikzentimeter bei der Kompression durch den äussern hydrostatischen Druck $\mathrm{P}$ erfährt, auszudrücken. $\varkappa$ ist definiert als Quotient aus der Volumverminderung pro Kubikzentimeter in den Druck, gemessen in Dynen pro Quadratzentimeter, also

$$
\Omega=\left(\mathrm{v}_{2}-\mathrm{v}_{1}\right) / \mathrm{v}_{1}=\boldsymbol{x} \cdot \mathrm{P} .
$$

Die bei der Kompression eines Kubikzentimeters geleistete Arbeit $\mathrm{W}$ ist somit

$$
\mathrm{W}=\int_{0}^{\Omega} \mathrm{Pd} \Omega=\frac{1}{\varkappa} \int_{0}^{\Omega} \Omega \mathrm{d} \Omega=\frac{\Omega^{2}}{2 \varkappa}
$$

Ist a der Abstand zweier Molekeln vor der Kompression und wieder wie oben $\Delta$ die relative mittlere Verschiebung der Molekeln bei der Volumverminderung $\Omega$, so ist, weil $\Omega$ klein gegen die Einheit, $\Omega=3 \Delta /$ a, wodurch nunmehr W sich ergibt zu

$$
\mathrm{W}=\frac{9 \Delta^{2}}{2 \varkappa \mathrm{a}^{2}} \cdot . \cdot
$$


Führt man noch an Stelle der Winkelgeschwindigkeit $\omega=$ $2 \pi \nu$ die Tourenzahl $\nu$ in Gleichung 1 a) ein, und setzt die beiden Ausdrücke für $\mathrm{W}$ einander gleich, so finden wir die Frequenz $\nu$ als Funktion des Kompressionsmoduls und des Molekelabstands a, also des wirklichen Molekularvolums $\mathrm{a}^{\mathbf{3}} \mathbf{z u}$

$$
\nu=\frac{3}{2 \sqrt{2} \pi} \cdot \sqrt{\frac{\mathrm{a}}{\mathrm{m} \cdot x}} \cdot \ldots . \quad \text {. . . }
$$

wobei wir von der. Beziehung $\mathrm{n}=\mathrm{a}^{-3}$ Gebrauch machen.

Ist $\mathrm{N}$ die Anzahl der Molekel im Mol, und führen wir weiter das Molekularvolum $\mathrm{V}=\mathrm{Nm} / \varrho=\mathrm{M} / \varrho$ ein, wobei $\varrho$ das spezifische Gewicht des Probekörpers bedeutet, so berechnet sich die Frequenz $\nu$ durch Einsetzen dieser Werte in 3) zu

$$
\boldsymbol{y}=\frac{3 \mathrm{~N}^{1 / 3}}{2 \sqrt{2} \pi} \cdot \mathrm{M}^{-1 / 3} \cdot \varrho^{-1 / 6} \cdot x^{-1 / 2} \quad . \quad \cdot \text {. }
$$

Setzt man für $\mathrm{N}$ den von Planck aus der Theorie der Hohlraum-Strahlung gewonnenen Wert $\mathrm{N}=6,175 \cdot 10^{23}$ ein, so wird schliesslich auf Grund der hier gegebenen Voraussetzungen die auch für die spezifische Wärme massgebende ultrarote Frequenz sich ergeben zu

$$
\nu=\frac{3,226 \cdot 10^{7}}{M^{1 / 3} Q^{1 / 6} \sqrt{x}}
$$

Wir erhalten also Tourenzahlen von der Grössenordnung $10^{12}$, welchen bei Nichtleitern Reststrahlen von den bei Steinsalz und Sylvin beobachteten Wellenlängen der Grössenordnung nach entsprechen. Um den Vergleich mit der Erfahrung zu erleichtern, führen wir an Stelle der Tourenzahl $\nu$ diejenige Wellenlänge $\lambda=$ c/ $\nu$ ein, welche den entsprechenden Reststrahlen im Vakuum zukommen würde, wobei $\mathrm{c}=3 \cdot 10^{10}$ die Lichtgeschwindigkeit im Vakuum bezeichnen soll. Gleichung 4) geht dadurch über in

$$
\lambda=\frac{2 \cdot \sqrt{2} \pi \mathrm{c}}{3 \mathrm{~N}^{1 / 3}} \cdot \mathrm{M}^{1 / 3} Q^{1 / 6} \sqrt{x}=0,930 \cdot 10^{3} \cdot \mathrm{M}^{1 / 3} Q^{1 / 6} \sqrt{x} .
$$

Unsere Gleichung ist somit bis auf einen Faktor von der Grössenordnung Eins identisch mit der von Einstein ${ }^{1}$ ) gegebenen Beziehung

$$
\lambda=\frac{2 \pi}{\sqrt{6}}\left(\frac{6}{\pi}\right)^{1 / 2} \cdot \frac{\mathrm{c}}{\mathrm{N}^{1 / 3}} \cdot \mathrm{M}^{1 / 3} \varrho^{1 / 6} \sqrt{x}=1,08 \cdot 10^{3} \cdot \mathrm{M}^{1 / 3} \cdot \varrho^{1 / 6} \cdot \sqrt{\varkappa}
$$

1) W. [4] 34, 170 (1911). 
obschon letztere unter der Voraussetzung, dass elastische Kräfte die schwingende Molekel in ihre Gleichgewichtslage zurücktreiben, hergeleitet ist, also unter denkbar andern Voraussetzungen als hier, wo ausschliesslich mit einem Zuwachs an kinetischer Rotationsenergie durch die Kompression gerechnet wurde.

Ohne deswegen etwa prinzipiell entscheiden zu wollen, welchen Ausgangshypothesen der Vorzug zu geben sei, schien es mir dennoch interessant zu untersuchen; ob die nach Einstein mit dem Faktor 1,08 oder nach unserer Herleitung mit dem um etwa $14 \%$ kleineren Faktor 0,93 berechneten Wellenlängen besser mit der Erfahrung, das heisst mit den aus der Quantentheorie der spezifischen Wärmen (Einstein ${ }^{1}$ ), Nernst und Lindemann ${ }^{2}$ ), $\left.D_{\text {Debye }}{ }^{3}\right)$ u. A.) berechneten oder bei Nichtleitern mit den nach der Methode der Reststrahlen gemessenen extremen ultraroten Wellenlängen übereinstimmen. Die folgende Tabelle erlaubt diese Frage $\mathrm{zu}$ entscheiden. Die erste Kolonne enthälţ die von Einstein selbst aus den Beobachtungen Grüneisens ${ }^{4}$ ) über die elastische Kompressibilität für eine Serie von reinsten Metallen berechneten Wellenlängen. Die gewählte Einheit ist $\mathrm{cm} \cdot 10^{-4}$. Da Einstein die übrigen von ihm zur Berechnung verwendeten Daten nicht angibt, habe ich die von ihm zur Berechnung verwendeten Werte der Wellenlängen, um dennoch einen einwandfreien Vergleich beider Formeln durchführen zu können, durch Multiplikation mit dem Quotienten 0,930/1,08 auf die neue Formel reduziert. Diese Werte, die man natiurlich auch direkt erhalten müsste, sobald man in unsere Formel dieselben Werte der Dichten und Atomgewichte, welche Einstein ${ }^{5}$ ) benutzt hat, einsetzt, gibt Kolonne 2 in Tabelle I.

Die dritte Kolonne ermöglicht den Vergleich mit der Erfahrung, und zwar sind hier ausschliesslich ${ }^{6}$ ) die aus den spezifischen Wärmen bei tiefen Temperaturen, also auf Grund der Abweichungen vom Dulong-Petit'schen Gesetz berechneten Werte der ultraroten Eigenschwingungen aufgenommen. Ich habe auch hier absichtlich die Darstellung nach Wellenlängen und nicht nach den unvorstellbar hohen Frequenzen $10^{12}$ gewählt. Die Werte

1) W. [4] 22, 185 (1907). $\quad$ 2) Z. El. Ch. 17, 817 (1911). 3) W. [4] 39, 789 (1912). 4) W. [4] 25, 848 (1908). 5) W. [4] 34, 170 (1911).

6) Der Wert für Palladium ist von Benedicks aus dem Schmelzpunkt berechnet. 
für $\mathrm{Al}, \mathrm{Gu}, \mathrm{Hg}$ und $\mathrm{Pb}$ sind der Arbeit Nernst und Lindernann's ${ }^{1}$ ), die übrigen einer Arbeit von C. Benedicks ${ }^{2}$ ) entnommen.

Tabelle I.

\begin{tabular}{|c|c|c|c|}
\hline & $\lambda$ Einstein & $\lambda$ Bernoulli & $\lambda_{\mathrm{Cp} .}$ \\
\hline Aluminium & 45 & 38,7 & 36,0 \\
\hline Nickel . & 45 & 38,7 & 37,5 \\
\hline Eisen & 46 & 39,6 & 35,7 \\
\hline Kupfer . & 53 & 45,6 & 45,5 \\
\hline Palladium & 58 & 49,9 & 48,4 \\
\hline Platin . & 66 & 56,8 & 56,6 \\
\hline Silber. & 73 & 62,9 & 66,0 \\
\hline Gold . & 79 & 70,1 & 70,1 \\
\hline Zinn $\left.{ }^{3}\right)$ & 102 & 87,8 & 80,0 \\
\hline Blei. & 135 & 116,2 & 153,6 \\
\hline
\end{tabular}

Da die Übereinstimmung zwischen der zweiten Kolonne und der dritten Kolonne zweifellos viel besser ist, als zwischen der ersten und der dritten, ist der neue Koëffizient (Gleichung 4a) dem frühern überlegen. Dabei ist die Übereinstimmung eine so hervorragend gute, dass wohl von jetzt an die aus der Kompressibilität, also aus der Volumelastizität berechneten Wellenlängen und Frequenzen, soweit es sich um Materialien handelt, die eine einwandfreie Bestimmung der elastischen Daten erlauben, als ebenso sicher gelten dürfen, wie die nach andern Methoden bestimmten. Interessant ist zweifellos, dass wir weder über die Art der Abhängigkeit der kinetischen Energie von der Temperatur, noch über die Grösse des Trägheitsmoments, also die Form der rotierenden Molekel oder Atome irgend welche Hypothesen haben einführen müssen.

Die folgende Tabelle II gibt einige Beispiele für die umgekehrte Aufgabe, die Berechnung von $x$ aus bekannten Schwingungszahlen.

1) Ber. Preuss. Akad. 12, 494 (1911). 9) W. [4] 42, 155 (1913).

3) Grüneisen hat später W. [4] 33, 1262 (1910) für Zinn die Kompressibilität nach 2 verschiedenen Methoden zu 1,9 und 3,1 und für Blei zu 2,0 und 3,2 gefunden. Bei diesen plastischen Materialien ist also $*$ noch wenig sicher. 
Tabelle II.

\begin{tabular}{|l|l|l|l|l|l|}
\hline & $\beta v$ & $\nu \cdot 10^{-12}$ & $\lambda$ & $x$ ber. & $x$ gef. \\
\hline \hline $\mathrm{Al}$ & 405 & 8,33 & 36,04 & 1,213 & 1,31 \\
$\mathrm{Ni}$ & - & 8,0 & 37,50 & $\mathbf{0 , 5 3 1}$ & 0,57 \\
$\mathrm{Fe}$ & - & 8,4 & 35,71 & 0,508 & 0,62 \\
$\mathrm{Cu}$ & 321 & 6,598 & 45,47 & 0,732 & 0,73 \\
$\mathrm{Ag}$ & 222 & $\mathbf{4 , 5 4 6}$ & 66,04 & 1,016 & 0,92 \\
$\mathrm{~Pb}$ & 95 & 1,953 & $\mathbf{1 5 3 , 6 4}$ & $\mathbf{3 , 4 6 9}$ & 3,2 \\
\hline
\end{tabular}

Wir gehen nun zur Berechnung der Atomwärmen aus der Volumelastizität über."

Liegt für eine einzige beliebige Temperatur eine genaue direkte Bestimmung der Volumelastizität, also der Konstanten $\varkappa$ vor, oder lässt sich die elastische Kompressibilität aus zwei andern elastischen Konstanten für das betreffende chemische Element hinreichend genau berechnen, so sollte der aus der elastischen Materialkonstanten $\varkappa$ unter Benützung unseres neuen Koëffizienten nach Gleichung 4a) berechnete Wert für die spezifische ultrarote Wellenlänge $\lambda$ oder die daraus abgeleitete Schwingungszahl $\nu$ ausreichen, um daraus die Atomwärmen und somit auch die Abweichungen vom Dulong-Petit'schen Gesetz für jede beliebige Temperatur zu berechnen. Über die Art der Abhängigkeit der Atomwärmen von der Temperatur lässt sich naturgemäss aus unserer rein kinetischen Ableitung von Gleichung 4a) ebensowenig irgend etwas aussagen, wie nach andern Theorieen, wenn nicht in Anlehnung an die Erfahrung willkürlich eine bestimmte Art der Abhängigkeit der Atomwärme von der absoluten Temperatur $\mathrm{T}$ und der ultraroten Eigenschwingung $\nu$ vorausgesetzt wird.

Wir machen nunmehr die Annahme, dass sich die Atomwärmen bei tiefen Temperaturen durch die von Debye ${ }^{1}$ ) angegebene Funktion von $v$ und $\mathrm{T}$ darstellen lasse.

Für die Metalle Aluminium, Blei und Kupfer habe ich nach Gleichung 4a) also mit Hilfe unseres neuen Koëffizienten aus den von Grüneisen ${ }^{2}$ ) in der Physikalisch-Technischen Reichsanstalt ermittelten Werten der kubischen Kompressibilität für

1) 1. c. S. 797 .

2) W. [4] 25, $848(1908)$ und $33,1262(1910)$. 
diese drei Metalle') die Eigenwellenlängen und die zugehörigen Schwingungszahlen berechnet. Daraus habe ich dann weiter mit Hilfe der Tabellen für die Debye'sche Funktion, welche wir Nernst $^{2}$ ) verdanken, die Atomwärmen unter der Voraussetzung, dass es sich um einatomige Elemente handelt, für alle diejenigen mittleren und tiefen Temperaturen neu berechnet, für welche experimentelle Bestimmungen vorlagen. Die Resultate dieser Rechnung sind in Tabelle III zusammengestellt. Für höhere Temperaturen ist die Umrechnung der spezifischen Wärmen auf konstanten Druck unerlässlich. Die dazu benützten Differenzen $\mathrm{G}_{\mathrm{p}}-\mathrm{G}_{\mathrm{v}}$ habe ich jedoch nicht aus Kompressibilität $\varkappa$ und Ausdehnungskoëffizient neu berechnet, was ja nach dem zweiten Hauptsatz der Thermodynamik hier mit aller Strenge möglich wäre. Ich habe dieselben vielmehr aus den von Nernst und Lindemann mitgeteilten und in Jellinek's ${ }^{3}$ ) trefflichem Werke über "Thermodynamik der Gasreaktionen" ausführlich reproduzierten Werten für $\mathrm{C}_{\mathrm{p}}$ und $\mathrm{C}_{\mathrm{v}}$ zurückgerechnet, um jede Willkür in der Wahl der zur Rechnung benützten Ausdehnungskoëffizienten auszuschliessen.

Tabelle III.

Aluminium.

\begin{tabular}{|c|c|c|c|}
\hline \multicolumn{2}{|c|}{$x=1,31$} & \multicolumn{2}{|c|}{$\beta \nu=389,9$} \\
\hline $\mathrm{T}$ & $\mathrm{Cr}$ ber. & & beob. \\
\hline 32,4 & 0,298 & 0,25 & Nemist \\
\hline 31,5 & 0.37 & 0,33 & n \\
\hline 83,6 & 2,57 & 2,41 & $"$ \\
\hline 86,0 & 2,68 & 2,59 & $"$ \\
\hline $88 ; 3$ & 2,79 & 2,62 & . \\
\hline
\end{tabular}

Bles.

$$
x=3,2 \quad \beta \nu=98,9
$$

\begin{tabular}{|c|c|c|c|c|c|c|c|}
\hline $\mathrm{T}$ & Gr her. & $(C p-C v)$ & Cp ber. & Cp & beob. & \multicolumn{2}{|c|}{ Cp beob. } \\
\hline 23,0 & $2,7 \check{5}$ & 0,01 & 2,76 & 2,96 & Neritst & 3,06 & lingh Onnes \\
\hline 28,3 & 3,45 & 0,01 & 3,46 & 3,92 & $n$ & 3,65 & $n$ \\
\hline 36,8 & 4,95 & 0,02 & 4,27 & 4,40 & $"$ & 4,48 & $"$ \\
\hline 38,1 & 4,31 & 0,02 & 4,33 & 4,45 & $n$ & 4,52 & $"$ \\
\hline 85,5 & 5,57 & 0,08 & 5,65 & 5,65 & " & 5,70 & $n$ \\
\hline 90,2 & 5,62 & 0,08 & 5,69 & 5,71 & $n$ & & \\
\hline 200 & 5,88 & 0,22 & 6,10 & 6,13 & Koret & & \\
\hline 290 & 5,92 & 0,34 & 6,26 & 6,33 & Gaede & & \\
\hline 332 & 5,93 & 0,38 & 6,31 & 6,41 & Magnus & & \\
\hline 409 & 5,94 & 0,46 & 6,40 & 6,61 & $"$ & & \\
\hline
\end{tabular}

1) Für Silbern ist leider $x$ noch nicht sicher genug bekannt.

2) W. Nerrast: ,Die Gundlagen des neuen Wirmesatzes". Halle (1908).

3) Letipzig (1913). 
Kupfer.

\begin{tabular}{|c|c|c|c|c|c|c|c|}
\hline$T$ & Cp ber. & $\mathrm{Cp} \mathrm{I}$ & beob. & $\mathrm{T}$ & Cp ber. & Cp beob. & Beobachter \\
\hline 23,5 & 0,182 & 0.223 & 3 Nerrest & 137 & 4,66 & 4,57 & Koref \\
\hline 27,7 & 0,299 & 0,324 & $n$ & 234 & 5,53 & $5, \overline{5} 9$ & , , \\
\hline 33,4 & 0,527 & 0,538 & $"$ & 290 & 5,76 & 5,79 & Gaede \\
\hline 87 & 3,31 & 3,33 & $"$ & 323 & 5,83 & 5,90 & Bartoli \\
\hline 88 & 3,34 & 3,38 & $n$ & 450 & 6,03 & 6,09 & Magnus \\
\hline
\end{tabular}

Die Durchsicht unserer Tabelle III lehrt, dass die Übereinstimmung zwischen den aus einem einzigen. Wert der Kompressibilität, also einer mechanischen Materialkonstanten berechneten Atomwärmen selbst bei extrem tiefen Temperaturen mit denjenigen Berechnungen für $\mathrm{C}_{\mathrm{p}}$, welche sich auf die aus dem Temperaturverlauf der spezifischen Wärmen entnommenen Werte der Eigenfrequenzen stützen, eine überraschend gute ist. Ungleich mehr will noch besagen, dass die Kö̈nzidenz zwischen unseren neuen berechneten Werten und der Erfahrung ganz besonders beim Kupfer, dessen elastische Daten im Gegensatz zum Blei nach verschiedenen Methoden. gut übereinstimmend ermittelt werden konnten, der Darstellung mit Hilfe der Eigenfrequenz aus den spezifischen Wärmen selbst, um nichts nachsteht.

Angesichts dieses Resultats darf ich vielleicht als historische Reminiszenz daran erinnern, dass Eirstein $\left.{ }^{1}\right)$ im Jahre 1911 in seiner grundlegenden Arbeit über "Eine Beziehung zwischen dem elastischen Verhalten und der spezifischen Wärme bei festen Körpern mit einatomigem Molekül"noch folgendes geschrieben hat: "An eine exakte Berechnung der Eigenschwingungsfrequenzen aus dèn elastischen Konstanten ist vorläufig nicht zu denken." Der neue Koëffizient, der eine Konsequenz unserer rein kinetischen Auffassung der Volumelastizität. darstellt, hat nummehr diese Aufgabe gelöst.

$$
\text { Zusammenfassung der Resultate: }
$$

1. Unter der Voraussetzung, dass die Atome oder die Molekeln f'ester Körper unter der Wirkung repulsiver Fernkräfte

j) W. [4] 34, 170 (1911). 
Eigenschwingungen ausführen, sind von Madelung, Sutherland, Einstein, Debye, Born und v. Kàrmàn u. A. quantitative Beziehungen zwischen Schwingungszahl und elastischer kubischer Kompressibilität abgeleitet worden. Im Gegensatz dazu gelingt es, die Einstein'sche Gleichung auch unter der Voraussetzung abzuleiten, dass die Kompressionsarbeit nur die kinetische Energie der Rotation der Molekel vermehrt, also nicht gegen repulsive molekulare Fernkräfte wirkt.

2. Ohne jede weitere willkürliche Annahme führt dies zu einem neuen Proportionalitätsfaktor jener Gleichung, welcher nunmehr eine erheblich bessere Übereinstimmung zwischen den aus der Kompressibilität und den aus spezifischen Wärmen berechneten Werten der ultraroten Eigenschwingungen ergibt, vorausgesetzt, dass das Material wirklich vollkommen elastisch ist.

3. Umgekehrt wird die Möglichkeit der Berechnung der Kompressibilität aus bekannten Werten der Schwingungszahlen an einigen Beispielen erwiesen, und ferner gezeigt, dass für die drei Metalle Kupfer, Aluminium und Blei die Abweichungen vom Dulong-Petit'schen Gesetz konform mit dem Experiment aus einer einzigen Kompressibilitätsmessung bei beliebiger Temperatur berechnet werden können.

Basel, Physikalisch-Chemische Anstalt der Universität. 